\title{
Influencia de la herbivoría sobre la interacción alga-coral en un arrecife coralino de bahía Capurganá, Caribe colombiano
}

\author{
Ibis Tarini López-Jiménez ${ }^{1}$, Lenin Flórez-Leiva ${ }^{1} \&$ Lizette Irene Quan-Young ${ }^{2}$ \\ 1. Ocean, Climate \& Environment Research Group, Programa de Oceanografía, Universidad de Antioquia, Antioquia, \\ Colombia; itlopezj@gmail.com, lennin.fl@gmail.com \\ 2. Grupo Biología CES, Facultad de Ciencias y Biotecnología, Universidad CES, Medellín, Colombia; \\ lquan@ces.edu.co
}

Recibido 19-III-2020. Corregido 17-IV-2020. Aceptado 06-V-2020.

\begin{abstract}
Influence of herbivory on coral-algal interaction in a coral reef, Capurganá Bay, Colombian Caribbean. Introduction: Coral reefs are among the most important and valuable ecosystems on the world. However, in recent decades they have been under siege by degradation processes reducing their coral cover and topographic complexity. The coral-algae competition is an important ecological process in the dynamics of coral reefs thus studying is pivotal for understanding the resilience in these systems. Objective: To determine the effect of herbivory on coral-algae competition under different scenarios (using a combination of descriptive and experimental approaches) and two climatic seasons in Capurganá Bay, Colombian Caribbean, in a marine conservation area (Hope Spot). Methods: Between July 2018 and April 2019, the role of herbivory was evaluated, performing an exclusion - no exclusion experiment in 48 coral individuals under different scenarios of interaction between Siderastrea siderea and its surrounding algae (treatments), half of the juvenile corals were covered with $2400 \mathrm{~cm}^{2}$ metal boxes fixed to the benthos. The treatments consisted of : $\mathrm{T} 1=$ No manipulation (control), $\mathrm{T} 2=$ Algae removal, $\mathrm{T} 3=$ Coral damage, $\mathrm{T} 4=$ Juveniles in contact with artificial algae, $\mathrm{T} 5=\mathrm{T} 2+\mathrm{T} 3$, $\mathrm{T} 6=\mathrm{T} 2+\mathrm{T} 4, \mathrm{~T} 7=\mathrm{T} 3+\mathrm{T} 4$ and $\mathrm{T} 8=\mathrm{T} 2+\mathrm{T} 3+\mathrm{T} 4$. Results: After nine months was found differences between scenarios with and without exclusion $(\mathrm{P}=0.006)$ and the climatic season $(\mathrm{P}=0.032)$, it was found that coral growth was higher when algae were removed in the presence of herbivores (Fisher's LSD, mean \pm SE $9.871 \pm$ 8.298) and the appearance of algae was greater inside the cages than outside them, while the competitive treatment did not show growth interference $(\mathrm{P}=0.155)$. Algae colonized juveniles when their tissue was damaged and herbivory was excluded (mean \pm SE $9.359 \pm 3.901$ ). There were differences in coral growth between seasons, being higher in the dry season (Average $0.0029 \pm \mathrm{SD} 0.0243 \mathrm{~cm}^{2} /$ day) than in the wet season (Average 0.0022 $\pm \mathrm{SD} 0.0161 \mathrm{~cm}^{2} /$ day). Conclusions: The coral growth depended on the presence of herbivores that regulate the growth, reproduction, and survival of algae; currently, this process is being interrupted as a result of overfishing in the reef ecosystems. However, some algae could be beneficial for juvenile corals when their abundance is large enough to protect them from fish predation and small enough not to significantly reduce their growth. These processes are decisive in these ecosystems, particularly in areas where it is relevant from an ecological, social, cultural and economic point of view, which makes these studies important for the correct implementation of integrated management in reef areas helping recovery, protection, and conservation of the reef promoting the decrease of herbivore fishing.
\end{abstract}

Key words: phase shifts; coral-algal competition; macroalgae; functional groups; Gulf of Urabá.

López-Jiménez, I.T., Flórez-Leiva, L., \& Quan-Young, L.I. (2020). Influencia de la herbivoría sobre la interacción alga-coral en un arrecife coralino de bahía Capurganá, Caribe colombiano. Revista de Biología Tropical, 68(3), 729-742. 
Los arrecifes coralinos son ecosistemas construidos por organismos marinos depositadores de carbonato de calcio, son de los ambientes más bio-diversos del planeta a pesar de ocupar solo $1 \%$ de la superficie marina (Gurney, Melbourne-Thomas, Geronimo, Aliño, \& Johnson, 2013). Estos ecosistemas también presentan alta producción primaria (Odum \& Odum, 1955; Dustan, 1999) además de un gran valor estético y comercial (servicios ecosistémicos valorados entre $5.5 \mathrm{y}$ 9.6 mil millones de dólares por año) (Odum, 1959; EPA, 2012), con una huella notable en las pesquerías y el turismo (Rendis, Acosta González, Hernández-Stefanoni, \& Arias-González, 2016; Hughes et al., 2017). Los arrecifes coralinos han sido impactados durante las últimas décadas por múltiples factores como la sobrepesca, eutrofización, enfermedades emergentes en herbívoros y corales, aumento de temperatura, deterioro de la calidad del agua, sedimentación, incremento de huracanes, ciclones, y eventos de blanqueamiento, colonización de algas sobre corales muertos, acidificación, etc. (Hughes et al., 2007; Díaz-Pulido, Gouezo, Tilbrook, Dove, \& Anthony, 2011). Estos factores han generado un reemplazo en la cobertura de organismos arrecifales por algas (Hughes et al., 2003; Vermeij et al., 2010), lo cual afecta la tasa de crecimiento coralina y reduce la biodiversidad marina (Venera-Pontón, Diaz-Pulido, McCook, \& Rangel-Campo, 2011; Cetz-Navarro, Quan-Young, \& Espinoza-Avalos, 2015).

De otro lado, la presencia y distribución espacial de los herbívoros en hábitats arrecifales depende de complejas interacciones entre distribución y número de refugios, abundancia de depredadores, densidad de competidores por territorio, y la estrategia que cada familia de herbívoros ha desarrollado (Ceccarelli, Jones, \& McCook, 2005; Jackson, Donovan, Cramer, \& Lam, 2014). Así los patrones de herbivoría generan heterogeneidad espacial mediante la regulación de las tasas de producción primaria, modificación de composición de la comunidad de las algas, la variación en la biomasa y la perturbación física de los hábitats (Augustine \& Frank, 2001; Vásquez, 2018). Por su parte, la sobrepesca local de herbívoros arrecifales limita la capacidad de los corales para resistir o recuperarse ante perturbaciones globales (Hughes et al., 2007; Schmitt, Holbrook, Davis, Brooks, \& Adam, 2019); ya que la pérdida de herbívoros en estos arrecifes comúnmente es seguida por la proliferación de macroalgas lo cual evita la recuperación coralina (Hughes et al., 2003; Folke et al., 2004). Sin embargo, la importancia de los procesos que median la proliferación de macroalgas y el cambio de fase de los arrecifes para lograr la recuperación de los corales aún se debate (Burkepile \& Hay, 2006; Smith, Hunter, \& Smith, 2010).

Un proceso importante que modula los arrecifes coralinos es la competencia algacoral, la cual juega un rol clave en los procesos de degradación arrecifal (Díaz-Pulido et al., 2011; Doropoulos \& Díaz-Pulido, 2013; Jackson et al., 2014). En un sistema arrecifal la competencia por el sustrato duro es intensa (Mejía-Niño \& Garzón-Ferreira, 2003) y por ello, los organismos sésiles suelen exhibir diversos mecanismos para colonizarlo, ocuparlo y mantenerlo (Márquez \& Díaz, 2005; Zea et al., 2007). Algunos crecen verticalmente para sombrear a sus vecinos u horizontalmente para recubrirlos y ahogarlos; algunas especies de coral tienen filamentos mesentéricos y tentáculos barredores con los cuales atacan a sus vecinos para defenderse y mantener libres sus límites (Zea et al., 2007); otros organismos emplean sustancias alelopáticas que matan el tejido vivo de sus vecinos (Thacker, Becerro, Lumbang, \& Paul, 1998; Mejía-Niño \& Garzón-Ferreira, 2003; Paul \& Puglisi, 2004).

Trabajos experimentales sobre interacciones entre algas, corales y herbivoría se han enfocado en la interacción natural entre peces herbívoros y algas (Stafford-Smith, 1992; Acosta-González, Rodríguez-Zaragoza, Hernández-Landa, \& Arias-González, 2013). Estos trabajos incluyen estudios que hacen énfasis en la caracterización e identificación de las especies de algas presentes en el ecosistema arrecifal (Cetz-Navarro, Espinoza-Avalos, Sentíes-Granados \& Quan-Young, 2008; CetzNavarro et al., 2015), otros sobre el cambio de 
fase alga-coral ocasionado por el crecimiento de algas epibiontes sobre corales pétreos (Contreras \& Ortegón-Aznar, 2012) y la valoración de interacciones entre corales y algas determinando que en el caso de los corales estas dependen de los mecanismos de competencia, de la eficacia y momento de acción de éstos; mientras que, en las algas, dependen del tipo y estrategia de crecimiento, y de la susceptibilidad a los herbívoros (Márquez \& Díaz, 2005). Por su parte, en el Caribe colombiano el proceso de liberación de espacio como consecuencia de la muerte de los corales ha facilitado la proliferación generalizada de las algas (Garzón-Ferreira, 1998; Arroyave, Bermúdez, \& Villada, 2014). Se ha demostrado que el incremento en la sedimentación, turbidez y contenido de nutrientes disueltos tienen un efecto negativo sobre la supervivencia y desarrollo de los corales, mientras que el aumento de estos favorece la proliferación de algas (Garzón-Ferreira, 1998).

En este trabajo se evaluó el efecto de la herbivoría sobre la competencia coral-alga bajo diferentes escenarios (empleando una combinación de enfoques descriptivos y experimentales) en los arrecifes coralinos de la Bahía Capurganá, Caribe colombiano.

\section{MATERIALES Y MÉTODOS}

Área de estudio: Este estudio se llevó a cabo en un arrecife de la costa de Capurganá (8³7’08.7” N \& 77¹9'53.6” W) ubicado en el Golfo de Urabá, Caribe colombiano (Apéndice digital 1) entre julio 2018 y abril 2019. La zona presenta temperatura promedio mensual de $27.3{ }^{\circ} \mathrm{C}$, humedad relativa de $83 \%$, y la precipitación oscila entre 4000 y $8000 \mathrm{~mm}$ anuales con un régimen de lluvias unimodal-biestacional y picos en abril y noviembre (IGAC, 2009). El fenómeno físico más influyente para la climatología intra-anual regional es el desplazamiento latitudinal de la Zona de Convergencia Intertropical (ZCIT), generando dos temporadas climáticas contrastantes, una seca y otra lluviosa (Poveda, 2004). En la temporada seca (de diciembre a marzo) predominan los vientos alisios desde el Noroeste con valores críticos superiores a $9 \mathrm{~m} / \mathrm{s}$, disminuye la precipitación y la temperatura $\left(27.70 \pm 0.06{ }^{\circ} \mathrm{C}\right)$, se incrementa la salinidad (33.81 $\pm 1.65 \mathrm{psu})$, la transparencia $(10.51 \mathrm{~m})$, la concentración de nutrientes inorgánicos (e.g. $\left.\mathrm{O}_{2}: 5.58 \pm 1.21 \mathrm{mg} / \mathrm{l}\right)$ (Chevillot, Molina, Giraldo, \& Molina, 1993) y la sedimentación disminuye, el oleaje y las corrientes son intensos presentando mares de leva y causando efectos negativos (e.g. fragmentación de colonias de corales, erosión) sobre algunas especies de coral (Andrade, 1993; Lonin \& Vásquez, 2005), se hace más frecuente la aparición de remolinos marinos y actividad de mesoescala que condicionan el transporte de sólidos suspendidos con la corriente. Mientras que en la temporada lluviosa de abril a noviembre, los vientos son débiles del sureste con velocidades no superiores a $7 \mathrm{~m} / \mathrm{s}$, procedentes del océano Pacífico y penetran por la cuenca del río Atrato desde la costa chocoana, aumenta la precipitación disminuyendo así la salinidad (34.41 $\pm 1.82 \mathrm{psu})$, aumenta la temperatura $\left(28.56 \pm 0.17^{\circ} \mathrm{C}\right)$, la carga de sedimentos en el agua, la turbidez $(9.4 \mathrm{~m})$ y la concentración de nutrientes. Debido a su forma semicerrada, el área de estudio está influenciada por la circulación de giros ciclónicos (Panamá - Colombia) y por la contracción costera del Golfo Darién, que fluye hacia el este formando un sistema de recirculación de agua (Andrade, 1993); y la dirección de la deriva no permite que los sedimentos del delta del Atrato se distribuyan a lo largo del golfo, por lo que el aporte de sedimentos se debe principalmente a las contribuciones biológicas de la costa chocoana (García-Valencia, 2007).

Diseño Experimental: La selección de las especies de coral y alga evaluadas se realizó durante el pre-muestreo. Al evidenciarse la presencia constante de Siderastrea siderea en el sitio de estudio y su facilidad para ser identificada en campo con las guías de Reyes \& Santodomingo (2002) y Hernández-Delgado (2009), se marcaron 48 individuos juveniles de esta especie de coral. Se buscaron especímenes sanos con tamaño entre $10-20 \mathrm{~cm}^{2}$, sin 
mordeduras o blanqueamiento visibles, a una profundidad entre 10-13 m, en condiciones de hábitat similares (grado de exposición y fisiografía similar) y con presencia de algas cercanas (entre 10-50 cm). Para evaluar la competencia coral-alga se tomó en cuenta la capacidad de cada organismo para colonizar el sustrato bajo dos parámetros: 1) cuando el sustrato está naturalmente ocupado por el presunto competidor y 2) cuando el presunto competidor ha sido intencionalmente herido o se le ha removido del sustrato. Se modificó el diseño metodológico de Venera-Pontón (2008) y Díaz-Pulido et al. (2011), implementando los tratamientos como se explica a continuación: $\mathrm{T} 1=$ No manipulación (control), T2= Remoción de algas, T3= Daño al tejido coralino,
T4= Juveniles en contacto con algas artificiales, T5= Remoción de algas + daño al tejido coralino, $\mathrm{T} 6=$ Remoción de algas + Juveniles en contacto con algas artificiales, $\mathrm{T} 7=$ Daño al tejido coralino + Juveniles en contacto con algas artificiales y $\mathrm{T} 8=$ Remoción de algas + Daño al tejido coralino + Juveniles en contacto con algas artificiales (Fig. 1). La medición del crecimiento de los corales se realizó con base al perímetro, diámetro máximo y longitud superficial máxima en cada individuo coralino, implementando el modelo de medición planteado por Venera-Pontón (2008). Las variaciones de estas medidas constituyeron un indicador del avance y la expansión de los juveniles coralinos en el sustrato, así como de la colonización algal sobre éstos.

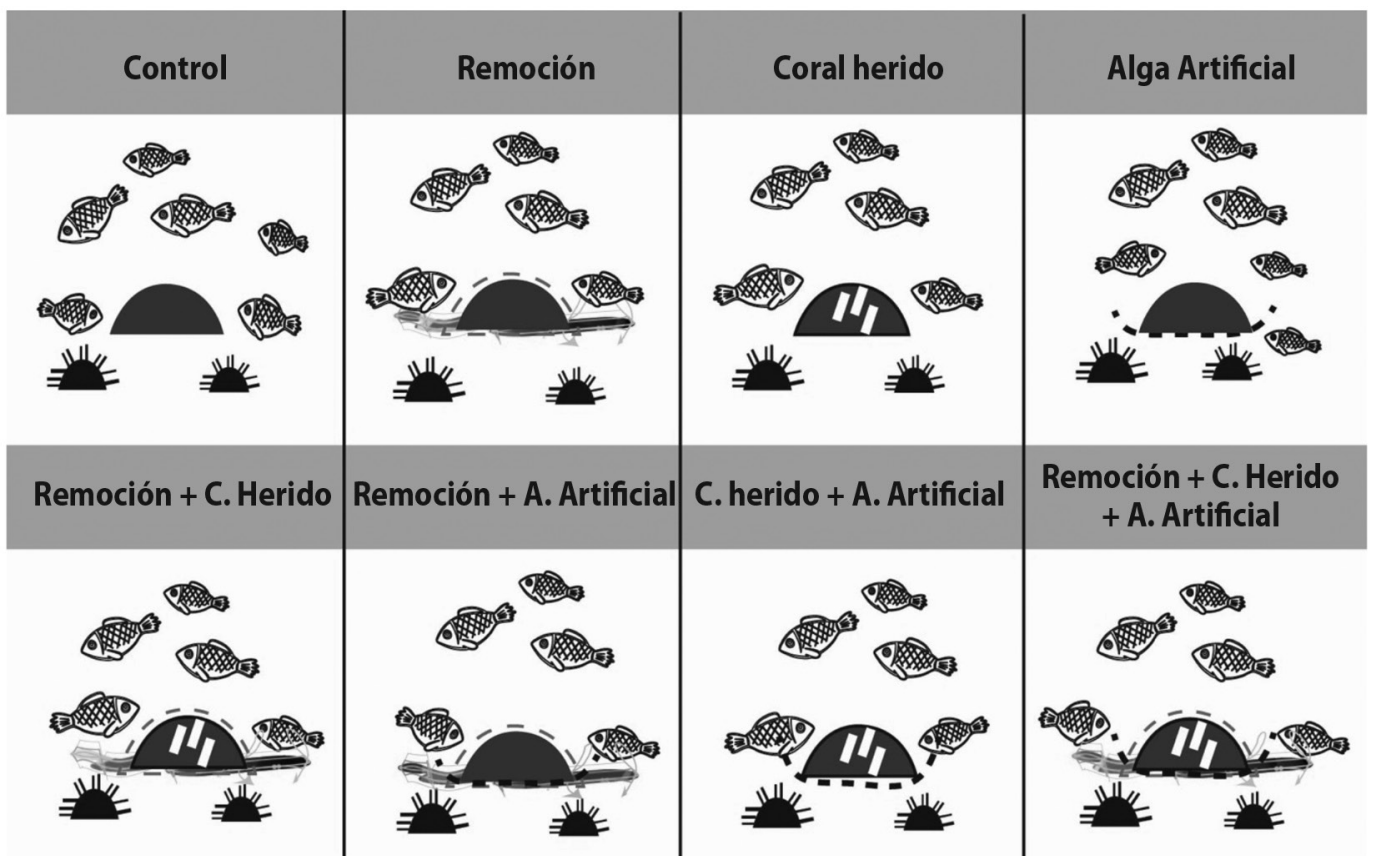

Fig. 1. Esquema gráfico del diseño experimental del efecto de herbivoría sobre la competencia coral-alga. Se plantean tres factores: 1. Herbivoría: (dos niveles: Con caja y sin caja). 2. Época climática (Húmeda y Seca). 3. Tratamientos de competencia (Ocho niveles: control, Remoción de algas, Daño coralino - herido, remoción + coral herido, Alga artificial, Algas + Alga artificial, Tejido Coralino + Alga artificial, Algas + Tejido Coralino + Alga artificial. Los rectángulos blancos indican las heridas en el coral, las líneas discontinuas grises la remoción de algas alrededor del coral, las líneas discontinuas negras representan las algas artificiales. Éste esquema fue replicado con jaulas y sin jaulas.

Fig. 1. Graphic diagram of the experimental design of the herbivory effect on coral-algae competition. Three factors are considered: 1. Herbivory: (two levels: With box and without box). 2. Climatic season (Wet and Dry). 3. Competitive treatments (Eight levels: control, Algae removal, Coral damage - wounded, removal + wounded coral, Artificial algae, Algae + Artificial algae, Coral tissue + Artificial algae, Algae + Coral tissue + Artificial algae. The white rectangles shows the wounds in the coral, the gray dashed lines the removal of algae around the coral, the black dashed lines represent the artificial algae. This scheme was replicated with cages and without cages. 
Para estimar el rol de la herbivoría, se realizó un segundo grupo dentro del experimento (bajo los mismos escenarios anteriormente mencionados) donde los juveniles se cubrieron con cajas metálicas con un área de $2400 \mathrm{~cm}^{2}$, las paredes fueron de malla plástica (ojo de malla de $1 \mathrm{~cm}^{2}$ ) fijas al bentos y protegidos de los peces. Para valorar si las habilidades competitivas de los organismos estudiados son afectadas por la estacionalidad, el experimento completo se realizó durante la época de lluvias (Julio a Noviembre) y repetido durante la época seca (Diciembre a Abril), con toma de datos bimensual.Durante todo el experimento se realizó censo visual de peces [siguiendo el protocolo AGRRA (2010) modificado para las condiciones del estudio], se tomaron fotografías y videos en toda el área de estudio. Se ignoraron todas las observaciones de peces juveniles $<15 \mathrm{~cm}$ de largo, debido a la dificultad de ser identificados con certeza a partir de las imágenes. La abundancia de las especies fue estimada a partir de la siguiente asignación: $\mathrm{A}=$ abundante $\left(>30\right.$ individuos $\left./ \mathrm{m}^{2}\right), \mathrm{C}=$ Común (entre 10 y 30 individuos $/ \mathrm{m}^{2}$ ) y $\mathrm{R}=$ Raro $\left(<5\right.$ individuos $\left./ \mathrm{m}^{2}\right)$. La identificación de los peces se realizó utilizando las descripciones de Humann (1997), Humann y Deloach (2002) y claves taxonómicas (Cervigón, 1994, 1996, 2005). Siguiendo el criterio de ViescaLobatón (2003), Robertson y Allen (2015) y Chasqui et al. (2017) se clasificaron las especies de peces por categoría ecológica según su tipo de alimentación (Carnívoros, Herbívoros, Omnívoros y filtradores). Los videos se realizaron con cámara GoPro 4 y Canon Powershot G7x mark II.

Finalmente, se empleó un diseño factorial, en el cual hubo cuatro factores con dos niveles: "herbivoría (Con y Sin caja)", Competencia: "Remoción (Con y Sin)", Algas artificiales (Con y Sin) y "Herida (Con y Sin)". Se realizaron tres réplicas de cada tratamiento.

Fase de laboratorio: Finalizada la remoción de algas, estas se depositaron inmediatamente en agua de mar con formol al $4 \%$ y fueron trasladadas al laboratorio para realizar el análisis e identificación taxonómica a nivel de especie y/o género usando claves especializadas como Parma, Pascual y Sar (1987), Littler y Littler (2000), Barreno-Rodríguez y PérezOrtega (2003) y León-Álvarez, Silva, Federico, Hernández-Almaraz \& León-Tejera (2017). Además, a cada especie o morfotipo le fue asignado un grupo morfo-funcional siguiendo la propuesta de Littler y Littler (2000). El nombre de las especies se asignó de acuerdo a Guiry y Guiry (2019).

Los efectos de la herbivoría sobre la competencia coral-algas se analizó utilizando un Análisis de Varianza (ANOVA) factorial de tres vías con: a) tratamientos de herbivoría (dos niveles: con y sin caja), b) época climática (dos niveles: Húmeda y Seca ), c) tratamientos de competencia (Ocho niveles: control, Remoción de algas, Daño coralino - herido, remoción de algas + coral herido, Alga artificial, remoción de algas + Alga artificial, Daño coralino + Alga artificial, remoción de algas + daño Coralino + Alga artificial). Se usaron los promedios estadísticos de dispersión (desviación estándar y error estándar). El modelo estadístico utilizó el área de cada colonia como variable respuesta a partir del cálculo de ésta utilizando los datos medidos (perímetro, diámetro máximo, y longitud superficial máxima) en cada individuo. Los supuestos de normalidad (test de ShapiroWilks) y homogeneidad de varianzas (test de Bartlett) se calcularon dentro de cada tratamiento. Para detectarlas diferencias mínimas significativas entre los tratamientos se empleó la prueba DMS (diferencia mínima significativa) de Fisher. El modelo se aplicó para un nivel de confianza del $95 \%$ en la determinación de los efectos estadísticamente significativos. Los análisis se realizaron utilizando los programas SPSS Versión 25 (IBM, 2018), Statgraphics Centurión XVI.I (Statpoint technologies, 2009) y R-Project (R Core Team, 2015).

\section{RESULTADOS}

La herbivoría y la época climática mostraron un efecto significativo sobre la variación del crecimiento coralino (Herbivoría $\mathrm{P}=0.006$; 
Época $\mathrm{P}=0.032$ ) (Apéndice Digital 2). Cuando los corales estaban descubiertos crecieron más que cuando estaban aislados con jaulas y la aparición de algas fue mayor dentro de las jaulas que fuera de ellas, mientras que el tratamiento de competencia no presentó interferencia en el crecimiento (Competencia $\mathrm{P}=0.155$ ) (Apéndice digital 2).

No se encontró diferencias significativas en los análisis de la interacción de los factores (herbivoría, época y competencia) $(\mathrm{P}=0.798)$ probablemente debido a un error tipo II. Hubo diferencias en el crecimiento del coral (área) entre las épocas, siendo mayor en la época seca $\left(\right.$ Media $=0.0029 \pm \mathrm{SD} 0.0243 \mathrm{~cm}^{2} /$ día $)$ que en la época húmeda (Media $0.0022 \pm \mathrm{SD}$ $0.0161 \mathrm{~cm}^{2} /$ día) (Fig. 2). La herbivoría presentó diferencias significativas $(\mathrm{P}=0.006)$ donde el crecimiento en el tratamiento con-exclusión fue menor (Media=11.221 \pm SD 5.966) que sin-exclusión (Media= 12.167 \pm SD 6.798).

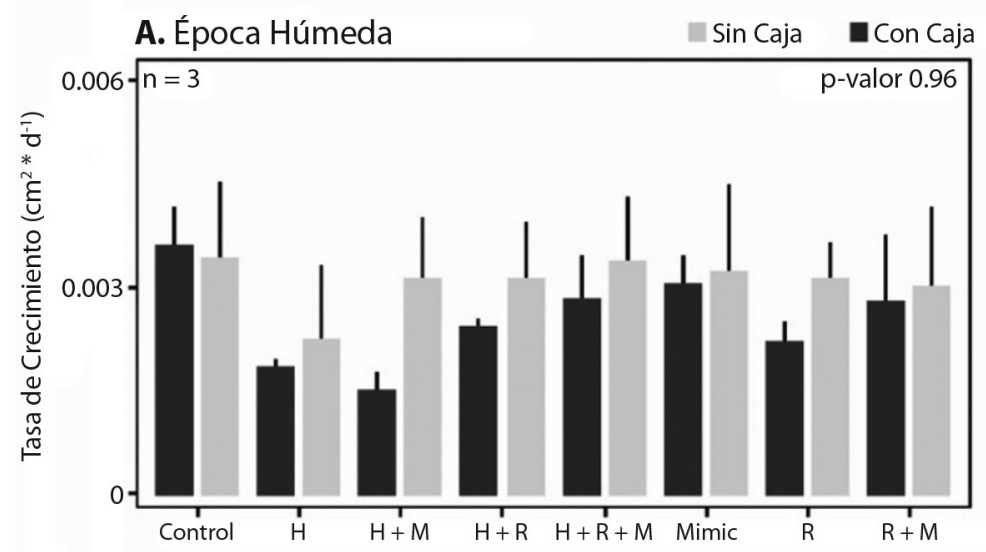

B. Época Seca

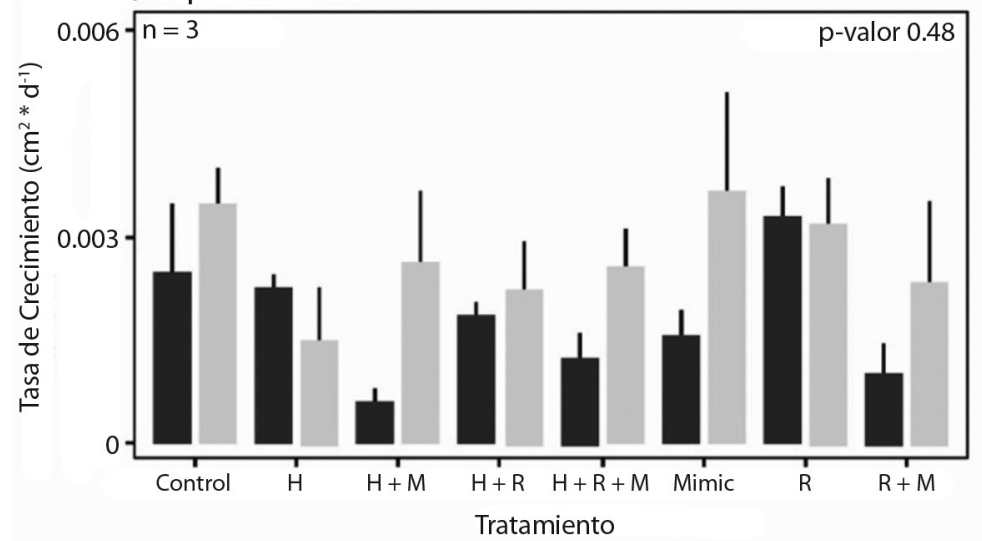

Fig. 2. Variación en el crecimiento de los individuos coralinos con exclusión y no exclusión de herbívoros a lo largo del experimento en la época húmeda y la época seca. (A) Época húmeda. (B) Época seca. Las barras negras representan la exclusión de herbívoros y las grises la no exclusión. $\mathrm{H}=$ Daño coralino - herido, $\mathrm{H}+\mathrm{M}=$ Daño coralino + Alga artificial, $\mathrm{H}+\mathrm{R}=$ Daño coralino + Remoción de algas, $\mathrm{H}+\mathrm{R}+\mathrm{M}=$ combinación de todos los tratamientos, Mimic $-\mathrm{M}=$ Alga Artificial, $\mathrm{R}=$ Remoción de algas, $\mathrm{R}+\mathrm{M}=$ Remoción + Alga artificial.

Fig. 2. Variation in the growth of coral with and without exclusion of herbivores throughout the experiment in the wet and dry seasons. (A) Wet season. (B) Dry season. The black bars represent the exclusion of herbivores and the gray bars the non-exclusion. $\mathbf{H}=$ Coral damage - wounded, $\mathbf{H}+\mathbf{M}=$ Coral damage + Artificial algae, $\mathbf{H}+\mathbf{R}=$ Coral damage + Algae removal, $\mathbf{H}+\mathbf{R}+\mathbf{M}=$ all treatments combined, Mimic, $\mathbf{M}=$ Artificial Algae, $\mathbf{R}=$ Algae removal, $\mathbf{R}+\mathbf{M}=$ Algal removal + Artificial algae. 
El experimento demostró que todos los individuos coralinos crecieron bajo las condiciones aplicadas partiendo de las condiciones iniciales de cada coral (Fig. 2). Independientemente del periodo climático, los juveniles coralinos crecieron más rápidamente (Media $0.00261 \pm \mathrm{SD} 0.00043 \mathrm{~cm}^{2} /$ día) cuando las algas fueron removidas (10 $\mathrm{cm}$ a la redonda) y los herbívoros no fueron excluidos, pero fueron colonizados por céspedes algales cuando sus tejidos fueron dañados (Fig. 2).

Los corales de los tratamientos T3 (daño al tejido coralino), T5 (remoción de algas + daño al tejido coralino) y T7 (daño al tejido coralino + juveniles con algas artificiales) durante ambas épocas no fueron colonizados por algas (ANOVA factorial, $\mathrm{P}>0.05$ ), aunque crecieron más rápidamente en la época húmeda (Media $=10.418 \pm$ SD 5.201) que en la época seca $($ Media $=11.623 \pm$ SD 5.603). Para el T1 (control) se encontró diferencia significativa entre épocas, siendo mayor el crecimiento en la época húmeda (31.03\% más) respecto a la época seca. Por su parte, el crecimiento de los juveniles coralinos heridos, fue mayor sin exclusión de herbívoros (aumento del $18.55 \%$, Media $=11.478 \pm$ SD 6.112) que con exclusión (Media $=9.359 \pm$ SD 3.90) en la época húmeda; mientras que en la época seca el mayor crecimiento fue evidenciado en los juveniles sin interacción con herbívoros (32.67 \% más, Media $=12.606 \pm$ SD 6.425) que en el tratamiento sin exclusión de herbívoros (Media $9.966 \pm$ SD 4.782). El crecimiento de los juveniles del T2 (remoción de algas) no presentó diferencias significativas (Apéndice digital 3), no obstante, en la época seca este tratamiento fue más beneficioso para el crecimiento coralino (con o sin exclusión de herbívoros) que, en la época húmeda, mostrando una respuesta positiva en el crecimiento de los juveniles con herbívoros (28.73\% más) que sin herbívoros $(\mathrm{P}=0.016, \mathrm{P}<0.05 ;$ Media $\pm \mathrm{DE}=9.871 \pm$ 8.298 y $9.229 \pm 5.901$ respectivamente).

Los resultados del experimento revelan que el tratamiento T6 (remoción de algas + juveniles con algas artificiales) en la época seca tuvo mayor crecimiento ( $55.82 \%$ más) con herbívoros respecto a la exclusión de herbívo$\operatorname{ros}($ Media $\pm \mathrm{DE}=16.312 \pm 8.600$ y $11.022 \pm$ 8.565 respectivamente), y en la época húmeda (DMS de Fisher, $7.48 \%$ ) el crecimiento de los corales con ausencia o presencia de herbívoros fue similar.

El efecto del T4 (juveniles con algas artificiales) influyó mayormente (57.01 \% más) en el crecimiento del coral en la época seca con presencia de herbívoros (Media= 17.244 \pm SD 7.785) que sin ellos (Media= $11.553 \pm$ SD 4.986), mientras que, en la época húmeda la respuesta fue mayormente homogénea (6.26 $\%$ ). Por su parte, el T8 (juveniles heridos con remoción de algas y algas artificiales) tuvo un comportamiento similar en ambas épocas (mayor crecimiento con herbívoros que sin ellos), el crecimiento en la época húmeda fue más homogéneo (Media \pm DE, $10.548 \pm 5.796$ con herbívoros y $9.748 \pm 2.431$ sin herbívoros) que en la época seca donde se evidencia una diferencia del $50.66 \%$ del crecimiento en el tratamiento con herbívoros respecto al tratamiento sin herbívoros (Media $\pm \mathrm{DE}=14.824 \pm$ 6.116 y $10.775 \pm 2.320$ respectivamente).

Los juveniles coralinos establecieron interacciones de contacto directo con algas de tapete (Cyanophyta, Rhodophyta, Chlorophyta) pero también fueron cubiertos por algas tipo carnosa y coralina. Se identificaron 75 morfoespecies de algas que fueron removidas de todos los juveniles de Siderastrea siderea (Apéndice digital 4), las cuales pertenecen a tres grupos morfofuncionales: dominadas por algas filamentosas de tapete (52.54\%), algas carnosas (35.59\%) y algas coralinas (11.86 $\%$ ) en menor abundancia. Las algas que fueron recolectadas tanto al inicio como al final del experimento presentaron una composición similar de especies en el cual la mayor presencia fue atribuida a los tapetes de algas. Sin embargo, se notó que en la época del inicio del experimento hubo mayor presencia de especies de algas de los géneros Cladophora, Ceramium y Chondria sp., mientras que al final Polysiphonia, Dyctiota y Lobophora fueron los más dominantes. 
Adicionalmente, por medio de censo visual en toda el área de estudio seleccionada, se identificaron un total de 33 especies de peces pertenecientes a 12 familias (Apéndice digital 5) donde las principales observadas corresponden a Labridae, Pomacentridae, Scaridae y Hemulidae. En general, los peces carnívoros, fueron los más abundantes durante todo el periodo de estudio (Apéndice digital 6), seguidos por los herbívoros (cuyas especies encontradas fueron comunes a lo largo del muestreo). Los peces territoriales (Pomacentridae) fueron el grupo más abundante de los registrados con $21.21 \%$ del total de especies registradas y el $25 \%$ del total de peces herbívoros siendo la especie Stegastes planifrons la más abundante del grupo. En cuanto a los herbívoros móviles (Scaridae y Acanthuridae) representaron el $24.24 \%$ de todas las especies de peces y el $66.66 \%$ del total de peces herbívoros observados. Las más abundantes de este grupo fueron: Acanthurus coeruleus, Sparisoma aurofrenatum y Scarus croicensis.

\section{DISCUSIÓN}

Aunque en el presente experimento no hubo un efecto significativo marcado en la competencia probablemente a un error tipo II, se ha notado que este tipo de interacciones está presente en la relación coral-alga (VeneraPontón, 2008), los juveniles coralinos crecieron más rápidamente en presencia de herbívoros y cuando las algas fueron removidas en ambos épocas climáticas; lo cual evidencia la existencia de competencia entre los juveniles coralinos de $S$. siderea y las algas arrecifales; como resultado de esto, la competencia expone como cada organismo involucrado (coral-alga) es reducido ante la presencia, abundancia o competidor (Birrell, McCook, Willis, \& Diaz-Pulido, 2008). Es pertinente mencionar que, no todas las especies de juveniles de coral están igualmente capacitadas para evitar el recubrimiento por parte de las algas, ya que esto depende de sus capacidades competitivas (Diaz-Pulido \& McCook, 2004; Nugues \& Bak, 2008). No obstante, y aunque las capacidades competitivas de los corales pueden mostrar a grandes rasgos características de las macroalgas que pueden ofrecer explicaciones más claras en casos particulares de dependencia entre las especies (coral o algas) y el modo como estas interactúan, es así como las macroalgas Lobophora sp., Dictyota sp., y Padina sp., debido a su rápido crecimiento pueden recubrir los corales (e.g. Agaricia sp., Acropora sp., Millepora sp.), reducir su asentamiento pero no matarlos (Box \& Mumby, 2007; Birrell, McCook, Willis, \& Harrington, 2008) e incluso en escenarios en que la competencia coral-alga se combina con altas concentraciones de dióxido de carbono en los arrecifes disminuyendo el tejido coralino y haciéndolos susceptibles a la proliferación de algas (Díaz-Pulido et al., 2011).

Algunos estudios han demostrado tasas de crecimiento en $S$. siderea parecidas a las encontradas en este trabajo para especies similares: tasas anuales entre $0,02+/-0,03$ y $0,03+/-0,05$ cm (Glynn, 1976; Weber \& White, 1977; Elahi \& Edmunds, 2007). Estudios han encontrado que el crecimiento y desarrollo de los corales adultos (e.g. Montastrea annularis, Porites sp.) son poco afectados por las algas (e.g. algas filamentosas de tapete), ya que estas son competitivamente inferiores a estos (Meesters, Pauchli, \& Bak 1997; Jompa \& McCook, 2003; Titlyanov, Yakovleva, \& Titlyanova, 2007); no obstante, en los juveniles coralinos el desarrollo se ve reducido por algas más dominantes (e.g. Dictyota sp., Lobophora sp. Sargassum sp.) capaces de sobrecrecerlos (Díaz- Pulido \& McCook, 2004; Box \& Mumby, 2007). Lo cual reafirma la teoría que los juveniles coralinos son más vulnerables a las algas en términos de competencia por espacio (Jompa \& McCook, 2003; Box \& Mumby, 2007; Birrell et al., 2008; Díaz-Pulido et al., 2011).

Aunque no se calcularon los incrementos en la cobertura de las algas pese a las elevadas tasas de crecimiento que tienen (Hay, 1981; McCook, 2001) y que los juveniles coralinos estudiados se mantuvieron en buen estado de salud, se pude pensar que $S$. siderea es relativamente fuerte en la competencia con los grupos de algas registrados [e.g. Lobophora el cual 
compite directamente con corales a distancias inferiores a $1 \mathrm{~cm}$ reduciendo su tasa de crecimiento (McCook, 2001)] en este experimento a corto plazo. La disminución del crecimiento de los juveniles coralinos como consecuencia del sobrecrecimiento de las algas bentónicas puede tener importantes implicaciones para la recuperación del ecosistema arrecifal en términos de los cambios de fase, ya que reduce la velocidad de crecimiento en ambientes actualmente dominados por algas y por tanto, la capacidad de recuperar la cobertura coralina perdida durante los cambios de fase (Díaz-Pulido et al., 2009).

De otro lado, el experimento evidenció también el efecto de la estacionalidad, ya que los periodos climáticos estudiados tienen una duración entre tres y cuatro meses (diciembre - abril y septiembre - noviembre) (GarzónFerreira, 1998; Rodríguez-Ramírez \& GarzónFerreira, 2003); evidenciando la influencia de los cambios ambientales y fisicoquimicos (temperatura, salinidad, nutrientes, transparencia) como determinantes en el crecimiento y desarrollo de los juveniles coralinos y de los ensamblajes algales del arrecife experimental, lo cual coincide con lo encontrado por Mejía-Niño (2001) donde se demostró que la competencia puede estar regida por la estacionalidad o las épocas climáticas (e.g zonas tropicales) con abundancias $H$. opuntia y el género Dictyota en épocas de transición (Mejía-Niño, 2001). Al observar las semejanzas del componente béntico (remoción al inicio y final del experimento), la interacción entre corales y macroalgas en los tratamientos son similares en cuanto a las especies y la frecuencia con que interactúan. Al parecer, las interacciones de $S$. siderea con macroalgas, particularmente con las algas filamentosas (e.g. Cladophora, Ceramium, Polysiphonia) las cuales siempre se encontraron en la periferia de las bases de las colonias son comunes y han sido reportados anteriormente (Lirman, 2001; Mejía-Niño, 2001). Los resultados obtenidos respecto a las macroalgas que interactúan con $S$. siderea coinciden con los trabajos realizados por Márquez y Díaz (2005) quienes reportaron que las macroalgas de los géneros Halimeda y Dictyota son las que se encuentran más frecuentemente en contacto con esta especie de coral.

En este estudio, los resultados de la exclusión de peces sugieren que existe un efecto crucial de la herbivoría sobre el crecimiento de $S$. siderea, ya que en el interior de algunas jaulas crecieron algas filamentosas, especialmente de los géneros Cladophora, Ceramium, Polysiphonia; esto es atribuído a que el crecimiento y la distribución de estos grupos algales dependen en gran parte del pastoreo (Steneck \& Testa, 1997; Jackson et al., 2014), el tipo de sustrato (Márquez, 1982), la intensidad de la luz (Markager \& Jensen, 1992), entre otras variables ambientales que determinan condiciones favorables o no para su desarrollo. Con base a esto se puede entender porque en este arrecife de la bahía Capurganá, predominen algunos grupos con mayor tolerancia ante efectos tensores (e.g. algas filamentosas de tapete) y por ende que sus interacciones sean más frecuentes, además de como la herbivoría juega un papel crucial en el crecimiento coralino, ya que eventualmente se observó en el interior de las jaulas a pequeños peces herbívoros de la familia Pomacentridae (Stegastes planifrons) y Acanthuridae (Acanthurus bahianus), los cuales ocasionalmente pueden hacer la labor de controlar el crecimiento de las algas filamentosas de tapete.

Por su parte, herbívoros de amplio espectro de forrajeo (Scarus croicensis y Scarus taeniopterus) pueden afectar positivamente a las colonias coralinas por el forrajeo sobre algas que crecen en la periferia de los individuos, aunque sus efectos positivos pueden ser limitados por el consumo accidental de coral (Miller \& Hay, 1998; Darling et al., 2017). Aunque el censo no mostró diferencias entre las épocas climáticas en la zona de estudio, es claro que, algunos peces cerca de las cajas no se registraron debido a la pequeña talla de estos lo cual limitó su identificación. Es posible que esta condición, sumada a la intensa actividad humana que existe normalmente, provoque que los peces estén más estresados y que las actividades territorialistas se vean aumentadas. 
Cabe mencionar, que el Caribe es muy susceptible a los cambios de fase (pasar de un ecosistema dominado por corales al predominio de algas) que otras regiones tropicales (Burkepile \& Hay, 2010; Roff \& Mumby, 2012), también se encuentra entre las regiones del trópico más pobres en especies en términos de riqueza de peces herbívoros, lo que explica la sensibilidad de esta región a la pérdida de especies y sabiendo que la Bahía Capurganá, presentan un alto grado de impacto antropogénico debido a diferentes factores como: el turismo, la descarga continental de ríos y los desagües de aguas residuales sin tratamiento que llegan al mar; esto hace que sea aparentemente una zona poco apta para el crecimiento corales (VivasAguas et al., 2014) y favorece el crecimiento de algas. Sin embargo, la presencia de las colonias de $S$. siderea involucradas en este estudio son atribuidas a su adaptación a tolerar condiciones estresantes como la sedimentación y la alta presencia de nutrientes (Sanders \& Baron-Szabo, 2005; Horta-Puga \& Carriquiry, 2008).

A la postre, se infiere que la reducción de la herbivoría, la presencia y sobrepoblación de macroalgas en el ecosistema coralino no son un indicador absoluto que determine el estado de la salud del arrecife coralino en Capurganá, sino a los diferentes instantes y eventos en el tiempo, independientes al momento en que se desarrolló el muestreo, pero que visto a la luz de los indicadores de salud arrecifal esto puede afectar los resultados de forma positiva o negativa (Rodríguez-Rincón, Navarrete-Ramírez, Gómez-López, \& Navas-Camacho, 2014), ya que los corales y las algas presentan variaciones y ciclos naturales que dependen completamente de factores propios de cada especie o de la época climática. Pese a que los arrecifes de la bahía Capurganá han sido considerados Hope Spot (Mission Blue, 2018), el turismo, la circulación de embarcaciones y la escorrentía (sedimentos, nutrientes y pesticidas) desde el continente han causado un mayor crecimiento de algas, acumulación de contaminantes en los sedimentos y especies marinas, reducción de la luz y corales sofocados. Estos cambios en la calidad del agua afectan principalmente la biodiversidad y la resistencia de los arrecifes, de tal manera que niveles más altos de clorofila y disminución en la transparencia del agua indican niveles más altos de concentración de contaminantes (sedimentos suspendidos, nitrógeno y fósforo) que conducen a más algas y menos diversidad de coral. En estas condiciones, las algas absorben y reducen las posibilidades de que nuevos corales duros se establezcan y crezcan. Es así como, en ciertos momentos del año, se presentan abundancias de algunas especies de macroalgas [e.g. bloom de cianobacterias registrado en el mes de octubre (año del inicio del muestreo) en la zona de estudio que cubrió gran parte del arrecife] sin que estas generen necesariamente muerte de las colonias coralinas.

Finalmente se puede concluir que, la presencia de coral abundante depende de altos niveles de herbivoría que regulan el crecimiento, reproducción y sobrevivencia de las algas y que éste proceso actualmente está siendo interrumpido como consecuencia de la sobrepesca en los ecosistemas arrecifales, por lo tanto, es importante destacar que la reducción de la herbivoría resultó en un crecimiento más rápido de algas, lo cual demuestra la importancia crítica de la herbivoría para el resultado de la interacción competitiva, de tal forma que se pudo demostrar experimentalmente que los céspedes de algas reducen la vulnerabilidad de los juveniles coralinos $S$. siderea a ser mordidos por peces, aunque esto no avala que la proliferación de alas sea favorable para la recuperación del ecosistema; es consecuencia de esto que este trabajo se realizó en esta área protegida, dada la importancia ecológica, social, cultural y económica de este ecosistema para la zona, ya que estudios de este tipo ayudan a la recuperación, protección y conservación del arrecife promoviendo la disminución de la pesca de herbívoros.

Declaración de ética: los autores declaran que todos están de acuerdo con esta publicación y que han hecho aportes que justifican su autoría; que no hay conflicto de interés de ningún tipo; y que han cumplido con todos los 
requisitos y procedimientos éticos y legales pertinentes. Todas las fuentes de financiamiento se detallan plena y claramente en la sección de agradecimientos. El respectivo documento legal firmado se encuentra en los archivos de la revista.

\section{RESUMEN}

Introducción: Los arrecifes coralinos están entre los ecosistemas más importantes y valiosos del planeta. Sin embargo, en las últimas décadas han sufrido procesos de degradación, reduciendo su cobertura coralina y complejidad topográfica. La competencia alga-coral es un proceso ecológico importante en la dinámica de los arrecifes coralinos y estudiarla permite entender la resiliencia en estos sistemas. Objetivo: determinar el efecto de la herbivoría sobre la competencia coral-alga bajo diferentes escenarios (empleando una combinación de enfoques descriptivos y experimentales) y dos épocas climáticas en Capurganá, Caribe colombiano (área de conservación marina, Hope Spot). Métodos: entre julio 2018 y abril 2019, se evaluó el rol de la herbivoría, mediante un experimento de exclusión - no exclusión en 48 individuos coralinos bajo diferentes escenarios de interacción entre Siderastrea siderea y sus algas circundantes (tratamientos), la mitad de los corales juveniles se cubrieron con cajas metálicas de $2400 \mathrm{~cm}^{2}$ fijas al bentos. Los tratamientos consistieron en: $\mathrm{T} 1=$ Control, T2= Remoción de algas, T3= Daño coralino, T4= Juveniles en contacto con algas artificiales, $\mathrm{T} 5=\mathrm{T} 2+\mathrm{T} 3$, $\mathrm{T} 6=\mathrm{T} 2+\mathrm{T} 4, \mathrm{~T} 7=\mathrm{T} 3+\mathrm{T} 4$ y $\mathrm{T} 8=\mathrm{T} 2+\mathrm{T} 3+\mathrm{T} 4$. Resultados: Después de un muestreo total de nueve meses, se encontró diferencias entre escenarios con y sin exclusión $(\mathrm{P}=0.006)$ y la época climática $(\mathrm{P}=0.032)$, el crecimiento coralino fue mayor cuando las algas fueron removidas en presencia de herbívoros (DMS de Fisher, mean \pm SE 9.871 $\pm 8.298)$ y la aparición de algas fue mayor dentro de las jaulas que fuera de ellas, mientras que el tratamiento de competencia no presentó interferencia en el crecimiento $(\mathrm{P}=0.155)$. Los juveniles fueron colonizados por algas cuando su tejido fue dañado y sin herbívoros (mean $\pm \mathrm{SE}$ $9.359 \pm 3.901)$. Hubo diferencias en el crecimiento coralino entre épocas (época seca: Media $0.0029 \pm \mathrm{SD} 0.0243 \mathrm{~cm}^{2} /$ día y época húmeda: Media $0.0022 \pm \mathrm{SD} 0.0161 \mathrm{~cm}^{2} /$ día). Conclusiones: Los resultados indican que el crecimiento coralino depende de la presencia de herbívoros que regulan el crecimiento, reproducción y supervivencia de las algas; actualmente este proceso está siendo interrumpido como consecuencia de la sobrepesca en estos ecosistemas, por lo cual, es importante destacar que la reducción de la herbivoría resultó en un crecimiento más rápido de algas, demostrando la importancia crítica de la herbivoría para el resultado de la interacción competitiva. Sin embargo, algunas algas podrían ser benéficas para los juveniles coralinos cuando su abundancia es lo suficientemente grande para protegerlos de la depredación por peces y suficientemente pequeña para no reducir considerablemente su crecimiento. Estos procesos son decisivos en estos ecosistemas, particularmente en zonas donde éste es relevante desde una perspectiva ecológica, social, cultural y económica, lo cual hace que estos estudios sean importantes para la correcta implementación de manejo integrado en zonas arrecifales ayudando a la recuperación, protección y conservación del arrecife, y promoviendo la disminución de la pesca de herbívoros.

Palabras clave: cambio de fase; competencia alga-coral; Macroalgas; grupos funcionales; Golfo de Urabá.

\section{REFERENCIAS}

Acosta-González, G., Rodríguez-Zaragoza, F.A., Hernández-Landa, R.C., \& Arias-González, J.E. (2013). Additive diversity partitioning of fish in a Caribbean coral reef undergoing shift transition. PLOS ONE, $8(6)$, e 65665.

Andrade, C. (1993). Análisis de la velocidad del viento en el mar Caribe. Boletín Cientifico CIOH, 13, 33-44.

Acevedo, L.A., Moreno, Y.B., \& Sierra, L.A.V. (2014). Impacto de la sedimentación en los corales de Islas del Rosario y San Bernardo, Colombia. Cuaderno Activa, (6), 133-141.

Augustine, D.J., \& Frank, D.A. (2001). Effects of migratory grazers on spatial heterogeneity of soil nitrogen properties in a grassland ecosystem. Ecology, 82 (11), 3149-3162.

Barreno Rodríguez, E., \& Pérez-Ortega, S. (2003). Líquenes de la reserva natural integral de Muniellos, Asturias. Asturias, España: KRK Ediciones.

Birrell, C.L., McCook, L.J., Willis, B.L., \& Harrington, L. (2008). Chemical effects of macroalgae on larval settlement of the broadcast spawning coral Acropora millepora. Marine Ecology Progress Series, 362, 129-137.

Birrell, C.L., McCook, L.J., Willis, B.L., \& Diaz-Pulido, G.A. (2008). Effects of benthic algae on the replenishment of corals and the implications for the resilience of coral reefs. Oceanography and Marine Biology, 46, 31 - 70. CRC Press.

Box, S.J., \& Mumby, P.J. (2007). Effect of macroalgal competition on growth and survival of juvenile Caribbean corals. Marine Ecology Progress Series, 342, 139-149.

Burkepile, D.E., \& Hay, M.E. (2006). Herbivore vs. nutrient control of marine primary producers: context-dependent effects. Ecology, 87(12), 3128-3139.

Burkepile, D.E., \& Hay, M.E. (2010). Impact of herbivore identity on algal succession and coral growth on a Caribbean reef. PLOS ONE, 5(1), e8963. 
Ceccarelli, D.M., Jones, G.P., \& McCook, L.J. (2005) Foragers versus farmers: contrasting effects of two behavioural groups of herbivores on coral reefs. Oecologia, 145(3), 445-453.

Ceccarelli, D.M., Jones, G.P., \& McCook, L.J. (2011) Interactions between herbivorous fish guilds and their influence on algal succession on a coastal coral reef. Journal of Experimental Marine Biology and Ecology, 399(1), 60-67.

Cervigón, F. (1994). Los peces marinos de Venezuela. Caracas, Venezuela: Fundación Científica Los Roques.

Cervigón, F. (1996). Los peces marinos de Venezuela. Caracas, Venezuela: Fundación Científica Los Roques.

Cervigón, F. (2005). La ictiofauna marina de Venezuela: una aproximación ecológica. Boletín del Instituto Oceanográfico de Venezuela, 44(1), 3-28.

Cetz-Navarro, N.P., Espinoza-Avalos, J., Sentíes-Granados, A., \& Quan-Young, L.I. (2008). Nuevos registros de macroalgas para el Atlántico mexicano y riqueza florística del Caribe mexicano. Hidrobiológica, 18(1), 11-19.

Cetz-Navarro, N.P., Quan-Young, L.I., \& Espinoza-Avalos, J. (2015). Morphological and community changes of turf algae in competition with corals. Scientific Reports, 5, 12814.

Chasqui, V., Polanco, L.A., Acero, A., Mejía-Falla, P.A., Navia, A., Zapata, L.A., \& Caldas, J.P. (2017). Libro rojo de peces marinos de Colombia. Instituto de Investigaciones Marinas y Costeras Invemar, Ministerio de Ambiente y Desarrollo Sostenible. Serie de Publicaciones Generales de INVEMAR, 93.

Chevillot, P., Molina, A., Giraldo, L., \& Molina, C. (1993). Estudio geológico e hidrológico del Golfo de Urabá. Boletín Cientifico $\mathrm{CIOH}, 14,79-89$.

Contreras, A.C., \& Ortegón-Aznar, I. (2012). Cambio de fase coral-algas en el arrecife de coral de Mahahual, en el Caribe Mexicano. Universidad Autónoma de Yucatán. Proceedings of the 64th Gulf and Caribbean Fisheries Institute October 31 - November 5, 2011. Puerto Morelos, México.

Darling, E.S., Graham, N.A., Januchowski-Hartley, F.A., Nash, K.L., Pratchett, M.S., \& Wilson, S.K. (2017). Relationships between structural complexity, coral traits, and reef fish assemblages. Coral Reefs, 36(2), 561-575.

Díaz-Pulido, G., \& McCook, L.J. (2004). Effects of live coral, epilithic algal communities and substrate type on algal recruitment. Coral Reefs, 23 (2), 225-233.

Díaz-Pulido, G., McCook, L.J., Dove, S., Berkelmans, R., Roff, G., Kline, D.I., \& Hoegh-Guldberg, O. (2009). Doom and boom on a resilient reef: climate change, algal overgrowth and coral recovery. PLOS ONE, 4(4), e5239.
Díaz-Pulido, G., Gouezo, M., Tilbrook, B., Dove, S., \& Anthony, K.R. (2011). High $\mathrm{CO}_{2}$ enhances the competitive strength of seaweeds over corals. Ecology Letters, 14(2), 156-162.

Doropoulos, C. \& Díaz-Pulido, G. (2013). High $\mathrm{CO}_{2}$ reduces the settlement of a spawning coral on three common species of crustose coralline algae. Marine Ecology Progress Series, 475, 93-99.

Dustan, P. (1999). Coral reefs under stress: sources of mortality in the Florida Keys. Natural Resources Forum, $23,147-15$

Elahi, R. \& Edmunds, P. J. (2007). Consequences of fission in the coral Siderastrea siderea: growth rates of small colonies and clonal input to population structure. Coral Reefs, 26(2), 271-276.

Folke, C., Carpenter, S., Walker, B., Scheffer, M., Elmqvist, T., Gunderson, L., \& Holling, C.S. (2004). Regime shifts, resilience, and biodiversity in ecosystem management. Annual Review of Ecology, Evolution, and Systematics 35, 557-581.

García-Valencia, C. (2007). Atlas del Golfo de Urabá: una mirada al Caribe de Antioquia y Chocó. Santa Marta, Colombia: Invemar.

Garzón-Ferreira, J. (1998). Bahía de Chengue, Parque Natural Tayrona, Colombia. CARICOMP-Caribbean Coral Reef, Seagrass and Mangrove Sites. Coastal Region and Small Islands Papers, 3, 115-125.

Glynn, P.W. (1976). Some physical and biological determinants of coral community structure in the eastern Pacific. Ecological Monographs, 46(4), 431-456.

Gurney, G.G., Melbourne-Thomas, J., Geronimo, R.C., Aliño, P.M., \& Johnson, C.R. (2013). Modelling coral reef futures to inform management: can reducing local-scale stressors conserve reefs under climate change? PLoS ONE, 8(11), e80137.

Guiry, M.D., \& Guiry, G.M. (2019). Algaebase. National University of Ireland. Galway, Ireland. World-wide electronic publication. http://www.algaebase.org

Hernández-Delgado, E.A., Shivlani, M., Sabat, A.M., \& Bruckner, A.W. (2009). Development of interdisciplinary criteria to identify priority candidate no-take marine protected areas in Puerto Rico Integration of ecosystem-based and community-based models. Silver Spring, Maryland, EE.UU.: Programa de Conservación de Arrecifes de Coral.

Hoey, A.S., \& Bellwood, D.R. (2011). Suppression of herbivory by macroalgal density: a critical feedback on coral reefs? Ecology letters, 14(3), 267-273.

Horta-Puga, G., \& Carriquiry, J. D. (2008). Growth of the hermatypic coral Montastraea cavernosa in the Veracruz Reef System. Ciencias Marinas, 34(1), 107-112.

Hughes, T.P., Baird, A.H, Bellwood, D.R, Card, M., Connolly, S.R, Folke, C., ... \& Lough, J.M. (2003). 
Climate change, human impacts, and the resilience of coral reefs. Science, 301(5635), 929-933.

Hughes, T.P., Rodrigues, M.J., Bellwood, D.R., Ceccarelli, D., Hoegh-Guldberg, O., McCook, L., ... \& Willis, B. (2007). Phase shifts, herbivory, and the resilience of coral reefs to climate change. Current Biology, 17(4), 360-365.

Hughes, T. P., Barnes, M. L., Bellwood, D. R., Cinner, J. E., Cumming, G. S., Jackson, J. B., ... \& Palumbi, S. R. (2017). Coral reefs in the Anthropocene. Nature, 546(7656), 82-90.

Humann, P. (1997). Reef Fish Identification. Jacksonville, Florida: New World Publications.

Humann, P., \& DeLoach, N. (2002). Reef Fish Identification Guide. Jacksonville, Florida: New World Publications.

IBM Corp. (2013). IBM SPSS Statistics para Windows, Versión 25.0. Armonk, NY: IBM Corp.

Jackson, J.B.C., Donovan, M.K., Cramer, K.L. \& Lam, V.V. (2014) Status and Trends of Caribbean Coral Reefs: 1970-2012. Gland, Switzerland: Global Coral Reef Monitoring Network, IUCN.

Jompa, J., \& McCook, L.J. (2003). Effects of competition and herbivory on interactions between a hard coral and a brown alga. Journal of Experimental Marine Biology and Ecology, 271(1), 25-39.

Lang, J. C., Marks, K. W., Kramer, P. A., Kramer, P. R., \& Ginsburg, R. N. (2010). AGRRA protocols version 5.4. Atlantic and Gulf Rapid Reef Assessment Program, Florida, USA. http://www.agrra.org

León Álvarez, D., Silva, C., Federico, C., Hernández Almaraz, P., \& León Tejera, H. (2017). Géneros de algas marinas tropicales de México I. Algas Verdes. Ciudad de México, México. Las prensas de Ciencias, Facultad de Ciencias, Universidad Nacional Autónoma De México.

Lirman, D. (2001). Competition between macroalgae and corals: effects of herbivore exclusion and increased algal biomass on coral survivorship and growth Coral reefs, 19(4), 392-399.

Littler, DS., \& Littler, M. M. (2000). Caribbean Reef Plants. Washington, D.C.: Offshore Graphics.

Lonin, S.A., Vásquez, A., \& Guillermo, J. (2005). Hidrodinámica y distribución de coniformes en el Golfo de Urabá. Boletín científico Centro de Investigaciones Oceanográficas e Hidrográficas, 23, 76-89.

Markager, S. \& Sand-Jensen, K. (1992). Light requirements and depth zonation of marine macroalgae. Marine Ecology-Progress Series, 88, 83-83.

Márquez, G. (1982). Los sistemas ecológicos marinos del sector adyacente a Santa Marta, Caribe colombiano I: Generalidades. Ecología Tropical, 2(1), 5-18.
Márquez, J.C., \& Díaz, J.M. (2005). Interacciones entre corales y macroalgas: dependencia de las especies involucradas. Boletín de Investigaciones Marinas y Costeras, 34, 227-242.

McCook, L. (2001). Competition between corals and algal turfs along a gradient of terrestrial influence in the nearshore central Great Barrier Reef. Coral Reefs, 19(4), 419-425.

Meesters, E.H., Pauchli, W., \& Bak, R.P. (1997). Predicting regeneration of physical damage on a reef-building coral by regeneration capacity and lesion shape. Marine Ecology Progress Series, 146, 91-99.

Mejía-Niño, N. (2001). Efecto e incidencia de las interacciones alga-coral en comunidades coralinas de dos bahías de la región de Santa Marta (Caribe colombiano) con distinto grado de influencia antropogénica. (Tesis de Bachillerato). Universidad de Bogotá Jorge Tadeo Lozano, Bogotá, Colombia.

Mejía-Niño, N. \& Garzón-Ferreira, J. (2003). Dynamics of algae-coral interactions in two bays of the Santa Marta region (Colombian Caribbean) with a different degree of anthropogenic influence. Boletín de Investigaciones Marinas y Costeras, 32(1), 243-261.

Miller, M.W., \& Hay, M.E. (1998). Effects of fish predation and seaweed competition on the survival and growth of corals. Oecologia, 113(2), 231-238.

Nugues, M.M., \& Bak, R.P.M. (2008). Long-term dynamics of the brown macroalga Lobophora variegata on deep reefs in Curacao. Coral Reefs, 27(2), 389-393.

Odum, H.T., \& Odum, E.P. (1955). Trophic structure and productivity of a windward coral reef community on Eniwetok Atoll. Ecological Monographs, 25(3), 291-320.

Odum, E.P. (1959). Fundamentals of Ecology. Philadelphia, Pennsylvania, EE. UU. WB Saunders Company.

Parma, A., Pascual, M., \& Sar, E. (1987). Clave para el reconocimiento de los géneros de algas macrófitas del intermareal rocoso bonaerense. Mar del Plata, Argentina. Serie técnica y didáctica-Facultad de Ciencias Naturales y Museo. Universidad Nacional de La Plata (Argentina).

Paul, V.J., \& Puglisi, M.P. (2004). Chemical mediation of interactions among marine organisms. Natural Product Reports, 21(1), 189-209.

Poveda, G. (2004). La hidroclimatología de Colombia: una síntesis desde la escala inter-decadal hasta la escala diurna. Revista Académica Colombiana de Ciencia, 28(107), 201-222.

R Core Team. (2015). R: A language and environment for statistical computing. R Foundation for Statistical Computing, Vienna, Austria. Recuperado de http,// www.R-project.org/ 
Rendis, A.M., Acosta González, G., Hernández-Stefanoni, J.L., \& Arias González, J.E. (2016). Quantifying the reefscape transformation of a coastal Caribbean coral reef during a phase shift and the associated coastal landscape change. Marine Ecology, 37(3), 697-710.

Reyes, J., \& Santodomingo, N. (2002). Manual de identificación CITES de invertebrados marinos de Colombia. Bogotá, Colombia. INVEMAR.

Robertson, D.R., \& Allen, G.R. (2015). Peces costeros del Pacífico Oriental Tropical: sistema de información en línea. Peces: Pacifico Oriental, Una guia para peces costeros del Pacifico Oriental Tropical. Balboa, República de Panamá. Instituto Smithsonian de Investigaciones Tropicales.

Rodríguez-Ramírez, A., \& Garzón-Ferreira, J. (2003). Monitoreo de arrecifes coralinos, pastos marinos y manglares en la Bahía de Chengue (Caribe colombiano): 1993-1999. Santa Marta, Colombia: Instituto de Investigaciones Marinas y Costeras José Benito Vives De Andreis.

Rodríguez-Rincón, A.M., Navarrete-Ramírez, S.M., Gómez-López, D.I., \& Navas-Camacho, R. (2014). Protocolo Indicador Condición Tendencia Áreas Coralinas (ICTAC). Indicadores de monitoreo biológico del Subsistema de Áreas Marinas Protegidas (SAMP). Santa Marta: Invemar, GEF y PNUD. Serie de Publicaciones Generales del Invemar, 66, 52 p.

Roff, G., \& Mumby, P.J. (2012). Global disparity in the resilience of coral reefs. Trends in Ecology and Evolution, 27(7), 404-413.

Sanders, D., \& Baron-Szabo, R.C. (2005). Scleractinian assemblages under sediment input: their characteristics and relation to the nutrient input concept Palaeogeography, Palaeoclimatology, Palaeoecology, 216(1-2), 139-181.

Schmitt, R.J., Holbrook, S.J., Davis, S.L., Brooks, A.J., \& Adam, T.C. (2019). Experimental support for alternative attractors on coral reefs. Proceedings of the National Academy of Sciences, 116(10), 4372-4381.

Smith, J.E., Hunter, C.L., \& Smith, C.M. (2010). The effects of top-down versus bottom-up control on benthic coral reef community structure. Oecologia, 163(2), 497-50.

Stafford-Smith, M.G. (1992). Mortality of the hard coral Leptoria phrygia under persistent sediment influx. Proceedings Seventh International Coral Reef Symposium, Guam, 1, 289-299.

Statgraphics Centurion, XVI. (2009). Statpoint technologies. INC. Warrenton, VA, USA.

Steneck, R.S., \& Testa, V. (1997). Are calcareous algae important to reefs today or in the past? Symposium summary. Proc $8^{\text {th }}$ International Coral Reef Symposio, 1, 685-688).

Thacker, R.W., Becerro, M.A., Lumbang, W.A., \& Paul, V.J. (1998). Allelopathic interactions between sponges on a tropical reef. Ecology, 79(5), 1740-1750.

Titlyanov, E. A., Yakovleva, I. M., \& Titlyanova, T. V. (2007). Interaction between benthic algae (Lyngbya bouillonii, Dictyota dichotoma) and scleractinian coral Porites lutea in direct contact. Journal of Experimental Marine Biology and Ecology, 342(2), 282-291.

Vásquez, C.E. (2018). Estructura de la comunidad coralina del arrecife de isla Lobos, Veracruz, México. (Tesis de pregrado). Universidad Autónoma De Baja California Sur, Área De Conocimiento De Ciencias Del Mar, Departamento De Biología Marina. La Paz, Baja California Sur, México.

Venera-Pontón, D.E., Díaz-Pulido, G., McCook, L.J., \& Rangel-Campo, A. (2011). Macroalgae reduce growth of juvenile corals but protect them from parrotfish damage. Marine Ecology Progress Series, $421,109-115$.

Vermeij, M.J., Van Moorselaar, I., Engelhard, S., Hörnlein, C., Vonk, S.M., \& Visser, P.M. (2010). The effects of nutrient enrichment and herbivore abundance on the ability of turf algae to overgrow coral in the Caribbean. PLoS ONE, 5(12), e14312.

Viesca-Lobatón, C. (2003). Cambios temporales en la estructura de la comunidad de peces de arrecifes rocosos en la parte sur-occidental del Golfo de California. (Tesis de Licenciatura). Universidad Autónoma De Baja California Sur, Área De Conocimiento De Ciencias Del Mar, Departamento De Biología Marina. La Paz, Baja California Sur, México.

Vivas Aguas, L.J., Sánchez, J., Cadavid, B., Betancourt, J., Quintero, M., Moreno, Y., ... \& Obando, P. (2014). Diagnóstico y Evaluación de la Calidad de las Aguas Marinas y Costeras del Caribe y Pacífico colombianos. Santa Marta, Colombia. Serie de Publicaciones Periódicas del Invemar.

Weber, J.N., \& White, E.W. (1977). Caribbean reef vorals Montastrea annularis and Montastrea cavernosa - Long-term rrowth data as determined by skeletal X-radiography. Reef Biota, 4, 171-179.

Zea, S., Duque, C., Castellanos, L., Chaves-Fonnegra, A., Márquez, J., \& Osorno, O. (2007). Impacto de esponjas excavadoras incrustantes en arrecifes del Caribe Colombiano II: mecanismos biológicos y químicos de agresión contra corales arrecifales. Santa Marta, Colombia. Universidad Nacional de Colo.bia, Informe Temático Divulgativo.

See Digital Appendix at: / Ver Apéndice digital en:

revistas.ucr.ac.cr 